\title{
Geometric Interpretation of the Origin of the Universe
}

\author{
Bohdan Lev \\ Bogolyubov Institute for Theoretical Physics, NAS of Ukraine, Metrolohichna 14-b, Kyiv, Ukraine \\ Email: bohdan.lev@gmail.com, bilev@bitp.kiev.ua
}

How to cite this paper: Lev, B. (2022) Geometric Interpretation of the Origin of the Universe. Journal of Modern Physics, 13, 89-99.

https://doi.org/10.4236/jmp.2022.132007

Received: January 7, 2022

Accepted: February 7, 2022

Published: February 10, 2022

Copyright $\odot 2022$ by author(s) and Scientific Research Publishing Inc. This work is licensed under the Creative Commons Attribution International License (CC BY 4.0).

http://creativecommons.org/licenses/by/4.0/

\begin{abstract}
An approach to the theory of geometrization of the Universe is proposed. The wave function of the Universe is represented by the Clifford number with the transfer rules that have the structure of the Dirac equation for any manifold. Solutions of this equation may be obtained in terms of the geometric interpretation. A new model is proposed that can explain the manifestation of the dark energy and dark matter in the Universe as a geometrical entity with a mechanism involving the spontaneous symmetry breaking.
\end{abstract}

\section{Keywords}

Clifford Algebra, Wave Function, Geometric Interpretation, Structural Equations

\section{Introduction}

The problem of origin of the Universe is far from being solved. Modern ideas (rather hypotheses) about the cause of the formation of the state of the universe suggest the instability of some fundamental scalar fields associated with the quantum nature of the matter [1]. The reasons and physical mechanism of the Universe origination remain open. In the paper [2] an approach was proposed to describe the causes and physical mechanism of the universe origination in terms of the first principles of statistical mechanics and quantum field theory. With this approach we can answer the question concerning the probable occurrence of an additional physical field, but nothing can be said about its geometric nature, except the assumption that everything has arose from a state of vacuum that corresponds to the lowest value of energy. In the case of spontaneous generation of an additional field in vacuum, the energy of the ground state of the "new" vacuum for the fields of different nature should be lower than the energy of the 
ground state of the "initial" vacuum. The interaction of the new field with fluctuations of a field of different nature ensures the decreasing of energy for the new state. There may occur a transition from the zero-field state to a state with the final spontaneously generated field. The new field interacts with the fluctuations of the vacuum, and in the presence of the nonlinear self-interaction caused by the fluctuations of different nature, a nonzero value of this field may occur.

We make an attempt to describe the fundamental field in terms of some physical entity, to derive the laws of its changes, and to find a mathematical apparatus that would describe these changes. The question arises about the geometric nature of this fundamental field. It may be scalar as well as have other geometric images. It is natural that its geometric characteristics are determined by the space that is created as the result of the distribution of matter. Without matter there is no point in talking about the geometry. In terms of physical characteristics, the most suitable at the moment is the Clifford number [2] [3]. The main idea of this paper is to describe the origination of the Universe in terms of the Clifford numbers and to find a probable explanation for the dark matter and energy, as well as to explain the observed meaning of the visible matter. To do this, we first focus on the basic properties of the Clifford algebra and show its advantages for the physical situation under consideration.

First of all we suggest that the spinor representation of the wave function of the universe as a quantum object is not very suitable for our case [4]. Cartan [5] showed that for the dimensional representation of spinors the complete linear coordinate transformation does not exist. Dirac spinors do not preserve the structure of the ring although they preserve the structure of the linear vector space. The allowed states are exhausted because it is impossible to calculate the behavior of the wave function during the parallel transfer and, moreover, it is impossible to determine the state of the ensemble of particles. In [6], a theorem is proved that states that associative algebra with the partition over the field of real numbers is real, complex, or Clifford algebra that uses the Clifford numbers and has the structure of the ring [7]. This is a vector space over the field of real numbers that is represented as an additive group where the multiplication of elements is distributive rather than commutative. This ring has ideals that may be obtained as a relevant projection on a specially selected element [7]. Such ideals are Dirac spinors in the standard approach. The representation of the Clifford algebra contains more information about physical properties than spinors. The geometric properties of the Clifford algebra may be naturally introduced into the theory of the Universe [1] [8] [9] [10] and employed to extend its physical meaning. We will try to show that the representation of Clifford's algebra best fits the description of the initial state of the Universe and provides more opportunities to explain both dark matter and baryon asymmetry.

As has been shown earlier [11] [12] [13] [14] [15], the application of the Clifford algebra contains all standard functions of the quantum mechanics and provides [3] [4] a unified basis for the physical knowledge including the theory of general relativity and electromagnetism. When we introduce the Clifford num- 
ber into the scheme of quantum mechanics [13], we should take into account the peculiarities of this formulation. In this case, we obtain a quantum-mechanics theory that considers only the algebraic structure and does not contain any specific requirements. The idea of this paper is to present the wave function of the universe by a geometric entity, i.e., the Clifford numbers, with the rules of transformation by the Dirac equation for any variety. The solutions of these equations may be obtained in terms of the geometric interpretation. Thus, the physical essence is described in terms of a geometric object with relevant transformation rules and the structure of the ring with respect to all algebraic operations. This makes it possible to highlight the contributions of the fields of different geometric nature in determining the energy and mass of the Universe.

\section{Clifford Algebra. Differentiable Manifold}

First from all we briefly describe the basic principles of Clifford's algebra with a view to their practical use. We use the basic idea [3] [4] [7], of the correspondence between matrices and basic elements of an algebra and thus define the space for the Clifford algebra. In the special theory of relativity the Dirac matrixs $\gamma_{\mu}$ acts as a unit vectors. An arbitrary linear combined product of these matrices has all the properties of the structure of the Clifford algebra with three complex units, starting with the time matrix $\gamma_{0}^{2}=1$ and three spatial matrices $\gamma_{\mu}^{2}=-1$. Therefore, we may reproduce any element belonging to the induced vector space as a direct sum of all probable tensor representations. In this case, an arbitrary function may be written in terms of the direct sum of a scalar, vector, bivector, trivector, and pseudo scalar that is given by

$$
\Psi=\psi_{0} \oplus \Psi_{\mu} \gamma_{\mu} \oplus \Psi_{\mu \nu} \gamma_{\mu} \gamma_{\nu} \oplus \Psi_{\mu \nu \lambda} \gamma_{\mu} \gamma_{\nu} \gamma_{\lambda} \oplus \Psi_{\mu \nu \lambda \rho} \gamma^{\mu} \gamma_{\mu} \gamma_{\nu} \gamma_{\lambda} \gamma_{\rho}
$$

When we change the direction of the basis vectors to the opposite, we obtain $\bar{\Psi}=\Psi_{s} \ominus \Psi_{v} \oplus \Psi_{b} \ominus \Psi_{t} \oplus \Psi_{p}$. Another element of symmetry is the change of multiplication of the basis vectors to the inverse order in the representation of Clifford numbers, which yields $\tilde{\Psi}=\Psi_{s} \oplus \Psi_{v} \odot \Psi_{b} \ominus \Psi_{t} \oplus \Psi_{p}$ We introduce the notation $i \equiv \gamma_{5} \equiv \gamma_{0} \gamma_{1} \gamma_{2} \gamma_{3} \quad$ (we denote the complex number as $\tilde{i}$ ) and then we have another symmetry element, i.e., the multiplication by $i$, presented as $i \Psi$ that is not equivalent to $\Psi i$. Having introduced the elements of symmetry, we need to propose a mathematical operation over the field of Clifford numbers. The direct sum of the tensor subspace may be given a ring structure by means of a direct tensor product in the symbolic notation, i.e., $\Psi \Psi=\Psi \cdot \Phi+\Psi \wedge \Phi$, where $\Psi \cdot \Phi$ is an inner product or convolution that decreases the number of basis vectors and $\Psi \wedge \Phi$ is an external product that increases the number of basis vectors. If each Clifford number is multiplied by a fixed matrix that has one column with one element and all other zeros, then we may obtain a Dirac spinor with four elements. This column may be used to reproduce the spinor representation of each Clifford number. There is a complete correspondence between the spinor column and the elements of the external algebra introduced here pre- 
viously.

In the next step we have to find the rule of comparison of two Clifford numbers at different points of the probable manifold [4]. To do this, we have to determine the change of the geometric object under the action of a complete linear group of coordinate transformations, i.e., the deformation of the coordinate system and the rule of parallel displacement on various probable manifolds. An arbitrary deformation of the coordinate system may be expressed in terms of deformations of the basis vectors $e_{\mu}=\gamma_{\mu} X$, where $X$ is the Clifford number that describes arbitrary changes in the basis (including arbitrary displacements and rotations) that do not violate its normalization, i.e., under the condition $\tilde{X} X=1$. It is not difficult to verify because $e_{m u}^{2}=\tilde{X} \gamma_{m u} X \tilde{X} \gamma_{m u} X=\gamma_{m u}^{2} \tilde{X} X=I$ and this does not violate the definition of the basis norm [7]. Now, for an arbitrary basis, we define at each point in the space a single complete linearly independent form that is a geometric entity that characterizes this manifold point. Such a geometric entity may be specified using

$$
\Psi=\Psi_{0} \oplus \Psi_{\mu} e_{\mu} \oplus \Psi_{\mu \nu} e_{\mu} e_{\nu} \oplus \Psi_{\mu \nu \lambda} e_{\mu} e_{\nu} e_{\lambda} \oplus \Psi_{\mu \nu \lambda \rho} e_{\mu} e_{\nu} e_{\lambda} e_{\rho}
$$

If this point of manifold is occupied by the matter, then its geometric characteristics may be described by the coefficients of this representation, including the coordinate basis $e_{\mu}=d x_{\mu}$. A product of arbitrary forms of this type is given by a similar form with new coefficients, thus providing the ring structure. This approach makes it possible to consider the mutual relation of fields of different physical nature [3] [16]. In what follows we may consider a new concept of the description of a particle and the characteristics of a manifold as a geometric entity.

Defining the characteristics of a manifold as a function of a point implies associating each point of the set with the Clifford number and its value. If this function is differentiated with respect to its argument, then we have to introduce a differentiation operation [3]. To determine the transfer operation on an arbitrary manifold, we have to determine the operator of derivative. This operation may be defined as $D=\gamma_{\mu} \frac{\partial}{\partial x_{\mu}}$, where $\frac{\partial}{\partial x_{\mu}}$ is associated with the change along the curves passing through a given point in space. The act of this operator at any Clifford number may be represented as

$$
D \Psi=D \cdot \Psi+D \wedge \Psi
$$

where $D \cdot \Psi$ and $D \wedge \Psi$ may be regarded as the "divergence" and "rotor" of the relevant Clifford number. According to the definition of the differentiated manifold, a single coordinate system is insufficient for covering a manifold whose topology differs from the topology of an open set in the Euclidean space

The structure of such a geometric construction should be supplemented by the correlation between the values of the transferred forms at different points of the manifold [3]. When assigning internal values to the characteristics of the manifold, we should introduce the transformation of Clifford numbers by changing the coordinate system. It may be identified by displaying the relevant Clifford numbers under the action of a certain group associated with the corres- 
ponding transformation. The conversion is possible if it is caused by any geometric characteristics changing the coordinate system accordingly, as well as by transforming the geometric objects. This requires the full use of the Clifford algebra as elements of the group of the internal vector space (group $S p(n)$ ) $X Y=Z$, where $X, Y, Z$ have similar preliminary representation. A certain group of transformations converts each Clifford number by the law $\Psi^{\prime}=\Psi X$, where $X$ determines the elements of the reflection of the Clifford algebra in our case and satisfies the condition $\tilde{X} X=1$. For this algebra, we may write the first structure equation that defines the covariant derivative [3] as given by:

$$
\Omega=d \Psi-\omega \Psi
$$

with the law of calibration transformation for the connectivity

$$
\omega^{\prime}=X \omega \tilde{X}+X d \tilde{X}
$$

for the conservation co-variant transformation according to the similar law $\omega^{\prime}=\omega X$. This equation is referred to as the first structure equation, but now it acquires the meaning in the Clifford algebra. In this case, an arbitrary Clifford number may always be reduced to a canonical form though the local deformations of the eigenbase become, however, unobservant because the Tetrude form $X d \tilde{X}$ corresponds to the second term of the calibration transformation. Then the second structure equation that defines the "curvature" form may be written as

$$
F=d \omega-\omega \omega
$$

with the law of transformation under the algebra being given by $F^{\prime}=X F \tilde{X}$. The transfer equation for the curvature tensor with the transformation law may be written in the form

$$
d F-F \omega+\omega F=J
$$

where $J$ is the flow form with the analogous general representation that complies the transformation $J^{\prime}=X J \tilde{X}$. The equation thus obtained may be regarded as the field equation, its form is apparently similar to the analogous equation for the connectivity form obtained in Lie algebra [3] [4]. Those equations possess a more general character as their structure contains interrelation of the geometric characteristics whose tensor nature is different. In this presentation we may write the fourth structure equation that demonstrates the dependence between the covariant derivation and the curvature, i.e., $d \Omega-\Omega \omega+\Psi F=0$.

It is natural to assume that each elementary formation at an arbitrary point of the manifold may be described by a Clifford number. Then the wave function of the elementary formation is represented by a complete geometric object, i.e., the sum of probable direct forms of the induced space of the Clifford algebra. Moreover, by attributing a geometric interpretation to the wave function, we may obtain correct transfer rules for an arbitrary manifold [3] and new results related to the geometric nature of the wave function [7]. According to [7], each even Clifford number $\Psi=\bar{\Psi}$ under the condition $\Psi \tilde{\Psi} \neq 0$, in the Euclidean 
space may be presented in the canonical form, i.e., $\Psi=\{\rho(x) \exp (i \beta)\}^{\frac{1}{2}} X$, where $X \tilde{X}=1$ describes all coordinate transformations. It is clear that $\int \tilde{\Psi} \Psi d \tau$ is scalar and in the physical interpretation of this geometric entity it is rather evident inasmuch as $\rho(x)$ may be associated with the probability density of finding a particle in an arbitrary spatial point, and $\beta$ is the angle that determines the eigenvalue of a particle with positive or negative energy. We can take $\beta=0$ for the matter and $\beta=\pi$ for the antimatter. Thus it becomes possible to describe the intermediate states of the particle since the form of the wave function of an arbitrary particle ensemble is similar [10]. It is important that $\rho \equiv \tilde{\Psi} \Psi=\left(\Psi_{s}+\Psi_{p}\right)^{2}+\Psi_{b}^{2}$ are represented by the products of different tensor representations of the general type of the wave function that have different geometric interpretations and correspond not only to the scalar field but may have different physical origins of the fields of different nature. It is proved in the book [7] that the odd part of the general Clifford number may be presented as the even part multiplied by a separate element of this algebra and thus it is not difficult to manipulate with the full Clifford number. Now for the wave function as a geometric entity, we may write the first structure equation in the standard form, i.e.,

$$
d \Psi-\omega \Psi=m \Psi
$$

that formally reproduces the Dirac equation but has wider meaning than in the spinor representation. The question of describing the wave function as a geometric entity was considered earlier in the article [4]. Among these results, we indicate that the Dirac equation in the geometric representation in the general theory of relativity is nothing but the equation of transfer on an arbitrary manifold, therefore, its solution may be interpreted purely geometrically. Moreover, the geometric representation of the wave function yields other results that simply reveal the geometric nature of the wave function [17]. Next, these equations will be derived from the principle of least action in the geometric interpretation. The presented equations first of all solve the problem of transformation of a finite-dimensional representation of the wave function under the action of a complete linear group of coordinate transformations [3] [7].

\section{Geometrical Origin of the Universe}

Next we assume that the occurrence in the vacuum of the fundamental scalar field that is generated spontaneously and interacts with the fluctuations of all other fields may be associated with a phase transition that owes to the decrease of the vacuum ground state energy [2] [18]. Moreover, evolution of the Universe formed by the fluctuations of physical fields may be described in terms of the Clifford number $\Psi$ [4]. The probable stationary distributions of the fundamental field are generated by the multiplicative noise produced by the nonlinear interaction. After that, the standard cosmological model may be modified. The fundamental field in the form of all probable geometric representations interacts 
with fluctuations through the change of the parameter of coupling of the given field with vacuum. Such fluctuations may be considered as a source of the multiplicative vacuum noise. In this case, such noise not only changes the value of the field, but also changes the shape of the effective potential due to the changes in the state of the system. This effect, in turn, changes the conditions for the formation of bubbles of a new phase and determines the evolution of the Universe. The generator of this noise is the vacuum itself in the form of a wave function for each point of the manifold with the Planck size. This model differs from the known scenario of stochastic inflation of the universe [1] that takes into account the fluctuations of the fundamental field but disregards the fluctuations of the unstable vacuum due to the fluctuations in the coupling parameter. The internal fluctuations of the manifold generate the stochastic behavior of the system that may induce changes of its stationary state. The most significant point here is that now the fundamental field is described by the Clifford number rather than scalar and contains all the geometric characteristics of the space that may be born as the result of the emergence of the matter. Only the distribution of the matter can describe the space that arises.

We start with the assumption that phase transition from the "initial" vacuum with only fluctuations of different fields to a new state of vacuum generates a new non-zero fundamental field. This means that the presence of a new field makes the "new" vacuum different from the "primary" vacuum for any field of arbitrary geometric characteristics that may exist. The resulting field should reduce the energy of the "new" vacuum with respect to the energy of the "primary" vacuum. Therefore, the energy density of the ground state of the "new" vacuum may be supplied through $\varepsilon=\varepsilon_{v}-\frac{\mu_{0}^{2}}{2} \tilde{\Psi} \Psi$, where the second part is the field energy in the term associated with the wave function with the geometrical presentation in terms of the Clifford numbers; the coefficient $\mu_{0}^{2}$ describes the coupling of the new field and the "primary" vacuum, i.e., the self-consistent interaction of the new field with the probable fluctuations that may exist in the "primary" vacuum. Here we have to make two remarks. The first one concerns the decrease in the initial energy of the ground state with the appearance of the new field, and the second one is related to the coupling coefficient that is now positive and thus explanations of the appearance of such a sign used in the standard approach are not required. The energy of the new system may be presented in the form given by

$$
E=E_{v}-\int \frac{\mu_{0}^{2}}{2} \tilde{\Psi} \Psi \mathrm{d} \tau,
$$

If we want to describe the evolution of the system $\langle$ out $|\exp i H t| i n\rangle$, we still need to average all probable fluctuations with which the new field can interact. For this purpose it is sufficient to present the nonlinear coupling in the form $\mu_{0}^{2}=\mu^{2}+\xi$, where $\langle\xi(t) \xi(0)\rangle=\sigma^{2}$ and $\sigma^{2}$ is the dispersion of the coupling coefficient fluctuations which allows averaging over all possible fluctuations 


$$
\begin{aligned}
\langle\text { out }|\exp i H t| \text { in }\rangle & \sim \int D \Psi \int D \xi \exp i\left\{E_{v}-\frac{1}{2} \mu^{2} \tilde{\Psi} \Psi+\frac{1}{2} \xi \tilde{\Psi} \Psi+\frac{\xi^{2}}{\sigma^{2}}\right\} \\
& \sim \int D \Psi \exp i\left\{E_{v}-\frac{1}{2} \mu^{2} \tilde{\Psi} \Psi+\frac{\sigma^{2}}{4}(\tilde{\Psi} \Psi)^{2}\right\}
\end{aligned}
$$

This implies that we have a system with the effective energy (averaged over the fluctuations of the other field coupled with the wave function) given by

$$
E=E_{v}-\int\left[\frac{1}{2} \mu^{2} \tilde{\Psi} \Psi-\frac{\sigma^{2}}{4}(\tilde{\Psi} \Psi)^{2}\right] \mathrm{d} \tau
$$

where $V(\Psi)=-\frac{1}{2} \mu^{2} \tilde{\Psi} \Psi+\frac{\sigma^{2}}{4}(\tilde{\Psi} \Psi)^{2}$ is the well-known expression for the energy of the fundamental field [1] with the nonlinear coupling coefficient determined by the dispersion of fluctuations. This implies that with no new field $\Phi=0, E=E_{v}$ while for $\rho=\tilde{\Psi} \Psi=\frac{\mu^{2}}{\sigma^{2}}$ the expression for the effective ground state energy of the "new" vacuum reduces to $E=E_{v}-\frac{\mu^{4}}{4 \sigma^{2}} \tau$. The last relation suggests the conclusion that the energy of the "new" vacuum is lower than the energy of the primary vacuum, i.e., the phase transition results in the formation of a new vacuum ground state with non-zero additional field that has new geometric presentations. If $\sigma^{2}$ tends to infinity, then the energy of the new state tends to the initial energy of the ground state. The energy of the new state can vanish for $E_{v}=\frac{\mu^{4}}{4 \sigma^{2}}$. This relation may be applied to estimate the maximum dispersion of vacuum fluctuations. In addition, the effective potential can now be given in terms of the probability density of the material field $V(\rho)=-\frac{1}{2} \mu^{2} \rho+\frac{\sigma^{2}}{4} \rho^{2}$, which may be useful for the interpretation of different compositions of energy and matter as a result of spontaneous breaking symmetry. It should be noted that this is the total probability density of the material field, and whether it is "dark" depends on the tensor characteristics of the field in which we feel it. It may be invisible in the vector electromagnetic field but will definitely be felt in the gravitational and possibly in the fields of another tensor presentation.

\section{Geometrical Description Evolution of the Universe}

Now we can offer a slightly different manifestation of the birth of the universe based on the representation of its wave function in terms of geometric essence. What arises as a result of the birth of matter must contain a geometric image. From the point of view of geometry, only the distribution of matter can be interpreted. This role can be played by Clifford's number with the appropriate physical interpretation. An additional field is required for the emergence of matter, the spontaneous excitation of which leads to the emergence of elementary particles. In our case, such a field is the wave function $\Psi$ in terms of dif- 
ferent tensor representations, i.e., it has all probable tensor representations with the dimension of the space to be created. That is, the geometry is laid down from the very beginning in the characteristics of the point of the variety on which we describe it.

Having minimized the expression for the energy of the system 9 by independent functions $\Psi$ and $\tilde{\Psi}$, we obtain for the wave function in the homogeneous case the Gross-Pitaevskii equation with all the physical consequences for solving such an equation.

$$
\frac{\delta E}{\delta \tilde{\Psi}}=\left[-\mu^{2}+\sigma^{2}(\tilde{\Psi} \Psi)\right] \Psi=0
$$

Equations similar in content but richer in nature may be obtained from the dynamics of changes of the wave function in the geometric interpretation. To do this, we consider the dynamical action recorded for the wave function of the universe in the presence of matter. As has been mentioned earlier [3], the action in terms of the geometric invariant may be presented as

$$
S=\int d \tau\left\{\frac{1}{2}(F \tilde{F}+\tilde{F} F)+m \Psi \tilde{\Psi}\right\}
$$

The Lagrange multiplier $m$ takes into account the normalization condition for the wave function $\int d \tau\{\Psi \tilde{\Psi}\}=1$. The "general" curvature in the presentation of Clifford numbers takes the form; $F=d \Psi-\lambda \Psi \Psi$ where the coefficient $\lambda$ takes into account the dependence of the connectivity field on the wave function itself $\omega=\lambda \Psi$. Minimization of this functional yields an equation that is at the same time the second structure equation for the Clifford algebra, i.e.,

$$
d F-F \lambda \Psi+\tilde{\lambda} \Psi F=J
$$

for the change of the "curvature" under the parallel transfer under the influence of the full group of transformations of the coordinate system. In the homogeneous case $d \Psi=0$ such equation reduces to the above Gross-Pitaevskii equation: $\left[-\mu^{2}+\sigma^{2}(\tilde{\Psi} \Psi)\right] \Psi=0$ with $\mu^{2}=m$ and $\sigma^{2}=\tilde{\lambda} \dot{\lambda}$.

To apply this approach to the description of the universe, we have to make a natural assumption. The new vacuum contains nothing except the born formation. For this reason, all changes associated with the wave function are due only to its changes in the vacuum in its presence. Therefore, its behavior can be influenced by only one characteristic of the new vacuum, namely this wave function. In this case, the wave function itself acts as a field that changes its characteristics, or as a connectivity of the space with the new vacuum. The equation required for the wave function is natural in the form

$$
d \Psi-\lambda \Psi \Psi=F, d F-F \lambda \Psi+\lambda \Psi F=J
$$

when the first structure equation is at the same time the second structure equation for the "curvature" $F$. It is assumed that $\omega \sim \lambda \Psi$, which corresponds to our previous assumption where $\lambda$ determines the relationship of the wave function to manifold.

On the other hand, it is easy to verify that if we consider odd Clifford numbers 
under the assumption $\Psi_{\mu} \sim A_{\mu}$ and $\Psi_{\mu v \rho} \sim \Gamma_{\mu \nu \rho}$, then we simultaneously reproduce both Maxwell and Einstein equations for the curvature for different components of the last equation, provided that the covariant derivative of the even part $\Psi_{\mu v} \sim g_{\mu v}$ yields zero. As example

$F_{\mu v}=A_{\mu, v}-A_{v, \mu}+\rho A_{\mu} A_{v}+\lambda \Gamma_{\mu \nu \lambda, \lambda}+\Psi_{\mu \nu \rho \sigma} g_{\rho \sigma}+\lambda \Gamma_{\mu \nu \rho} A_{\rho}$ where $\left(1+\Psi_{s}\right) \Gamma_{\mu \nu \rho}=g_{v \lambda, \mu}+g_{\lambda \mu, v}+g_{\mu v, \rho}+\lambda g_{\mu v} A_{\rho}+g_{\rho v} A_{\mu}+\lambda g_{\rho \mu} A_{1}+\lambda \Psi_{\mu v \rho \sigma} A_{\sigma}$. All these elementary calculations are not given here because of their cumbersomeness [3].

It should be noted that the physical entity described by the wave function in the form of the Clifford number does not belong to certain quantum statistics and contains elements of both fermions and bosons. In the case of spontaneous symmetry breaking, a part of such an object turns into particles and a part remains a field, each of which corresponds to certain elements of symmetry. It is possible that in Clifford's algebra it is possible to write a more general relation which will take away information on a condition of the condensed part but at present it could not be found though it is possible to use the approach offered in article [19].

\section{Conclusions}

As the result, we propose a probable scenario for the formation of the universe. We assume that in the presence of a spontaneously generated fundamental field with different geometric presentation, the vacuum energy is lower than the ground state energy of the primary vacuum and that the ground field energy is influenced by its nonlinear interactions with the fluctuations of the physical fields of different nature. To avoid the problem of the influence of gravity on the evolution of the universe at the stage of spontaneous emergence of the fundamental field, we note that the energy of the primary vacuum is not contained in the Einstein equation and the evolution of the universe is determined only by the energy of the fundamental field.

Only the distribution of matter in turn determines the geometry. The birth of matter is determined only by a non-zero fundamental field that contains contributions of fields of different physical and thus geometric nature. The presentation of probability densities of material entities contains fields whose geometric characteristics do not overlap and therefore cannot be observed within the behavior of individual components. For example, the change in the electromagnetic field may not be affected by the field connectivity described by the tensor characteristic of the third rank. For our universe, the vacuum is different from the primary one and its state depends on the fundamental field that possesses different tensor representation. In addition to the scalar part of the fundamental field, there are fields of other tensor dimensions that may be involved in the influence of the dark matter.

\section{Conflicts of Interest}

The author declares no conflicts of interest regarding the publication of this paper. 


\section{References}

[1] Linde, A.D. (1990) Elementary Particle Physics and Inflationary Cosmology. Horwood Academic, Chan. https://doi.org/10.1201/9780367807788

[2] Lev, S.B. and Zagorodny, A.G. (2020) Journal of Modern Physics, 11, 502. https://doi.org/10.4236/jmp.2020.114032

[3] Lev, B.I. (1988) Modern Physics Letters, 3, 1025. ib.4, erratum, (1989).

[4] Lev. B.I. (2012) Journal of Modern Physics, 3, 709. https://doi.org/10.4236/jmp.2012.38096

[5] Cartan, E. (1938) Lecons sur ia theorie des spineurs. Actualites scientifiques et industries, Paris.

[6] Frobenius, G. (1878) Journal für die reine und angewandte Mathematik, 1878, 1. https://doi.org/10.1515/crelle-1878-18788403

[7] Kasanova, G. (1976) Vector algebra. Presses Universitaires de France, Paris.

[8] Hestenes, D. (2015) Space-Time Algebra. Springer International Publishing, Berlin. https://doi.org/10.1007/978-3-319-18413-5

[9] Hiley, B.J. and Callaghan, R.E. (2010) The Clifford Algebra Approach to Quantum Mechanics A: The Schroedinger and Pauli Particles. Corpus ID: 119265887.

[10] Doran, C. and Lasenby, A.N. (2003) Geometric Algebra for Physicist. Cambridge University Press, Cambridge. https://doi.org/10.1017/CBO9780511807497

[11] Conte, E. (1994) Physics Essays, 6, 4-10. https://doi.org/10.4006/1.3029089

[12] Conte, E. (1994) Physics Essays, 7, 14-20. https://doi.org/10.4006/1.3029160

[13] Conte, E. (2011) Electronic Journal of Theoretical Physics, 8, 109-126.

[14] Cini, M. (2006) Electronic Journal of Theoretical Physics (EJTP), 3, 1-10.

[15] Khrennikov, A. (2001) Journal of Physics A: Mathematical and General, 34, 99659981. https://doi.org/10.1088/0305-4470/34/47/304

[16] Benn, J.M. and Tucker, R.W. (1983) Physics Letters A, 130, 177. https://doi.org/10.1016/0370-2693(83)91037-7

[17] Klein, A.G. (1988) Physics B, 151, 44. https://doi.org/10.1016/0378-4363(88)90143-X

[18] Lev, B.I. and Zagorodny, A.G. (2020) Journal of Modern Physics, 11, 502. https://www.scirp.org/journal/jmp https://doi.org/10.4236/jmp.2020.114032

[19] Hackl, L., Guaita, T., Shi, T., Haegeman, J., Demler, E. and Cirac, J.I. (2020) SciPost Physics, 9, Article No. 48. https://doi.org/10.21468/SciPostPhys.9.4.048 